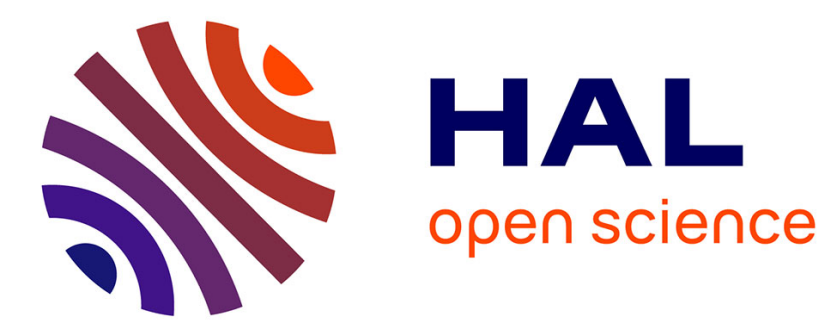

\title{
Optical Analysis of p-Type Surface Conductivity in Diamond with Slotted Photonic Crystals
}

Candice Blin, Xavier Checoury, Hugues Girard, Celine Gesset, Samuel Saada, Philippe Boucaud, Philippe Bergonzo

\section{- To cite this version:}

Candice Blin, Xavier Checoury, Hugues Girard, Celine Gesset, Samuel Saada, et al.. Optical Analysis of p-Type Surface Conductivity in Diamond with Slotted Photonic Crystals. Advanced Optical Materials, 2013, 1, pp.963 - 970. 10.1002/adom.201300331 . cea-01791383

HAL Id: cea-01791383

https://hal-cea.archives-ouvertes.fr/cea-01791383

Submitted on 11 Feb 2019

HAL is a multi-disciplinary open access archive for the deposit and dissemination of scientific research documents, whether they are published or not. The documents may come from teaching and research institutions in France or abroad, or from public or private research centers.
L'archive ouverte pluridisciplinaire HAL, est destinée au dépôt et à la diffusion de documents scientifiques de niveau recherche, publiés ou non, émanant des établissements d'enseignement et de recherche français ou étrangers, des laboratoires publics ou privés. 


\section{Advanced Optical Materials \\ Optical Analysis of p-Type Surface Conductivity in Diamond with Slotted Photonic Crystals \\ --Manuscript Draft--}

Manuscript Number:

Full Title:

Article Type:

Keywords:

Corresponding Author:

\section{Corresponding Author Secondary \\ Information:}

\section{Corresponding Author's Institution:}

Corresponding Author's Secondary Institution:

First Author:

First Author Secondary Information:

Order of Authors:

\begin{tabular}{|l|}
\hline Candice Blin \\
\hline Xavier CHECOURY \\
\hline Hugues GIRARD \\
\hline Céline GESSET \\
\hline Samuel SAADA \\
\hline Philippe BOUCAUD \\
\hline Philippe BERGONZO \\
\hline
\end{tabular}

Order of Authors Secondary Information:

Abstract:

Additional Information:

\section{Question}

Please submit a plain text version of your cover letter here.

adom.201300331R1 Crystals

Full Paper layer; Transfer doping.

Candice Blin

Candice Blin

\section{Response}

Optical Analysis of p-Type Surface Conductivity in Diamond with Slotted Photonic

CVD nanocrystalline diamond; photonic crystals; Nanocavities; Surface conductive

Diamond Sensors Laboratory

Gif sur Yvette, FRANCE

Diamond Sensors Laboratory

We report on the fabrication of two-dimensional slotted diamond-based photonic crystals (PhC) with $\mathrm{Q}$ factors up to 6500 and their optical characterization at $1550 \mathrm{~nm}$ in order to probe surface molecular modifications. In this study, we intentionally focus on the simplest surface modifications that can modify the diamond $\mathrm{PhC}$ optical properties, namely, hydrogenation and oxidation. We observed that, depending on the chemical surface termination, these diamond $\mathrm{PhCs}$ exhibit a strong modification of their spectral features. When the surface is tuned from oxidized to hydrogenated, a resonance wavelength shift of the cavity occurs and is accompanied by a decrease of the $Q$ factor. Moreover, we give experimental evidence that this phenomenon is reversible as the initial value of the $Q$ factor is recovered when the surface is reoxidized. We attribute this result to the sub-surface conductive layer that is due to transfer doping in hydrogenated diamond and which is absent from oxidized diamond. Thanks to 3D-FDTD simulations, we give an estimate of the effective refractive index of the surface conductive layer at $1.5 \mu \mathrm{m}$ as a function of its thickness. This result highlights the high sensitivity of slotted diamond $\mathrm{PhC}$ and the importance of surface control for biosensing with diamond.

Response for manuscript adom.201300331 "Optical Analysis of p-Type Surface Conductivity in Diamond with Slotted Photonic Crystals"Dear Dr Fuchs, We have 
Please note, if you are submitting a revision of your manuscript, there is an opportunity for you to provide your responses to the reviewers later; please do not add them to the cover letter. uploaded a revised manuscript referenced adom.201300331 entitled "Optical Analysis of p-Type Surface Conductivity in Diamond with Slotted Photonic Crystals". We thank the reviewers for their positive remarks as well as their helpful comments for improving the manuscript. All the comments of the reviewers have been answered. This letter includes the revised version of the manuscript with the main modifications emphasized in red color. Considering the improvements brought to the delivered manuscript, we hope that this revised version is now suitable for publication in Advanced Optical Materials. Sincerely yours, Candice Blin 
DOI: 10.1002/adom.201300331

Full paper

Title : Optical Analysis of p-Type Surface Conductivity in Diamond with Slotted Photonic Crystals

Authors : Candice Blin, Xavier Checoury *, Hugues Girard, Céline Gesset, Samuel Saada, Philippe Boucaud and Philippe Bergonzo *

C. Blin, Dr. H. Girard, C. Gesset, Dr. S. Saada, Dr. P. Bergonzo

CEA, LIST, Diamond Sensors Laboratory, Gif-sur-Yvette, F-91191, France

E-mail : philippe.bergonzo@,cea.fr

C. Blin, Prof. X. Checoury, Dr. P. Boucaud

IEF, CNRS Univ Paris Sud 11, Bâtiment 220, F-91405 Orsay Cedex, France

E-mail : xavier.checoury@,ief.u-psud.fr

Keywords: CVD nanocrystalline diamond, Photonic crystals, Nanocavities, Surface conductive layer, Transfer doping

We report on the fabrication of two-dimensional slotted diamond-based photonic crystals (PhC) with Q factors up to 6500 and their optical characterization at $1550 \mathrm{~nm}$ in order to probe surface molecular modifications. In this study, we intentionally focus on the simplest surface modifications that can modify the diamond $\mathrm{PhC}$ optical properties, namely, hydrogenation and oxidation. We observed that, depending on the chemical surface termination, these diamond PhCs exhibit a strong modification of their spectral features. When the surface is tuned from oxidized to hydrogenated, a resonance wavelength shift of the cavity occurs and is accompanied by a decrease of the Q factor. Moreover, we give experimental evidence that this phenomenon is reversible as the initial value of the Q factor is recovered when the surface is re-oxidized. We attribute this result to the sub-surface conductive layer that is due to transfer doping in hydrogenated diamond and which is absent from oxidized diamond. Thanks to 3D-FDTD simulations, we give an estimate of the effective refractive index of the surface conductive layer at $1.5 \mu \mathrm{m}$ as a function of its thickness. This result highlights 
Submitted to

the high sensitivity of slotted diamond $\mathrm{PhC}$ and the importance of surface control for biosensing with diamond.

\section{Introduction}

Using integrated photonic technologies, it is possible to fabricate very compact, high performing and low-cost chemical and biochemical sensors. Different kinds of integrated optical chemical sensors have been proposed over the years, from those based on surface plasmon resonance ${ }^{[1]}$ to micro-ring resonators. ${ }^{[2]} \mathrm{A}$ novel class of nanoscale biosensors which have been increasingly studied in recent years is photonic crystals (PhCs). Such devices can offer unique and high sensitivity for the detection of specific chemical and biological agents using small active volumes, thus making them promising structures for their use in medical or environmental applications. $\mathrm{PhCs}$ generally consist of a one- or two-dimensional periodically patterned dielectric structures where light can be confined to in-plane guided resonance modes in the slab. They are characterized by spectral features such as the resonance wavelength or the quality factor (Q). Most of the recent designs for cavities with high quality factors rely on the creation of an optical confinement along a W1 waveguide (one missing line of holes in a triangular lattice $\mathrm{PhC}$ ). This can be achieved for instance by a variation of the lattice constant ${ }^{[3]}$ or of the width of the waveguide..$^{[4]}$

Due to the tight confinement of the optical mode, PhCs are highly sensitive to local changes in their environment, ${ }^{[5]}$ such as the refractive index, which affect the resonance wavelength and the quality factor of the resonator. This shift of the resonance wavelength thus confers a sensing capacity to the cavity. Specificity in detection is brought by functionalizing the surface of the resonant cavity or waveguide, which can then be used to selectively capture a specific target molecule, in gas or liquid phase. ${ }^{[6,7]}$ The detection limit will thus be linked to the quality factor of the cavity, and so challenging $\mathrm{Q}$ factors are needed to obtain sufficiently sensitive sensors.

Nowadays, silicon is the reference material for such devices thanks to its optical properties and its well mastered nanostructuration. Recently, quality factor of a few tens of thousands were obtained 


\section{Submitted to \\ ADVANCED \\ MATERIALS}

in silicon PhC sensor cavities allowing to detect ultra-small refractive index changes at the surface of the sensors corresponding to an avidin bounded mass of approximately 100 ag. ${ }^{[6]}$ However, it has been reported that the quality factor of micro-disk resonators may vary from a factor of one to five when the silicon surface is changed from oxide to hydrogenated termination. ${ }^{[8]}$ This strong dependence was pointed out as one of the most critical challenge for future Si micro- and nanophotonic systems where extremely high Q-factors are usually required. Furthermore, $\mathrm{PhC}$ sensing devices will require a highly stable material to avoid any unsolicited perturbation of the surface, and surface stability of silicon may be a limitation, especially its functionalization which may display unstable properties in biological media. ${ }^{[9]}$

Among the dielectric materials available for $\mathrm{PhC}$ fabrication, diamond appears as a promising alternative to build such photonic-based devices: it is recognized to be an outstanding material in terms of mechanical properties, as well as in terms of bio-compatibility and surface functionalization. Diamond-based devices are now at an advanced state of development in the fields of electrochemistry, MEMS sensors, biomedical implants, protective coatings, etc. ${ }^{[10]}$ Diamond is currently emerging as a novel material for the development of photonics devices at a micrometric scale. ${ }^{[11]}$ It exhibits a refractive index of 2.4 , and diamond wide band gap offers transparency in a large spectral range from ultraviolet to far infrared spectral bands unlike Si which, in particular, is not transparent in the visible range. Indeed, these properties have already allowed the recent fabrication of photonic crystal cavities with quality factors reaching 3000 , not only on $3 \times 3 \mathrm{~mm}^{2}$ monocrystalline substrates, ${ }^{[12,13]}$ but also on polycrystalline diamond layers ${ }^{[14]}$ grown on two inches substrates, thus opening the route to large scale processing with standard nanotechnology techniques. Further, since diamond, unlike $\mathrm{Si}$, does not have a native oxide, it exhibits a stable surface which can be functionalized through a covalent carbon-carbon binding ${ }^{[15]}$ highly promising towards the fabrication of diamond-based photonic devices for chemical detection.

In this context, this work aims at evaluating how surface chemistry affects the optical properties of diamond-based photonic devices. If sophisticated and stable functionalizations are allowed on 
Submitted to

diamond surfaces, they rely on the stability of the initial surface terminations, usually either "hydrogenated" with C-H bonds, or "oxidized" with etheric, alcohol or carboxylic groups. These terminations are the prerequisite to any further functionalization, opening the route to specific grafting routes such a diazonium salts chemistry, peptidic linking, etc. Therefore, the effect of this basic surface chemistry on diamond optical properties has been studied in this work by the means of a specifically designed $\mathrm{PhC}$. We use a slotted photonic crystal $(\mathrm{shC})$ cavity to get a high sensitivity to surface refractive index change and then tune the diamond surface termination between hydrogenated and oxidized. As will be shown, a wavelength shift of the cavity occurs and is accompanied by a decrease of the Q factor when the surface chemistry is simply turned from oxidized to hydrogenated. This effect is reversible. These phenomena are attributed to the conductive layer known to exist from transfer doping on hydrogenated diamond surfaces, and we will below demonstrate how this feature will correlate with the behavior of such diamond-based photonic devices.

\section{Results and discussion}

\subsection{Simulation of slotted photonic crystals cavities}

A few types of PhC cavities have already been simulated and measured on diamond. ${ }^{[16]}$ The so called L3 or L7 cavities ${ }^{[12,17]}$ can present modes with quality factors up to a few thousands while modulated-width type cavities ${ }^{[14]}$ can theoretically reach Q factors above 1 million. So far there is no simulation result on slotted microcavities on diamond and only a few simulations have been reported on long slotted waveguides. ${ }^{[18]}$ The parameters that lead to the highest $\mathrm{Q}$ factors and smallest modal volumes for these slotted cavities may be different from the ones made in silicon due to the lower refractive index of diamond. In the following section, the best parameters to achieve high Q factors and small modal volumes are determined by using three dimensional finite differences in time domain simulations (3D-FDTD). The cavity design is similar to the one fabricated on silicon and described in ref. ${ }^{[19]}$. This design consists of a modulated width cavity 


\section{Submitted to \\ ADVANCED \\ MATERIALS}

similar to ref. ${ }^{[3]}$ with a slot (See Figure 1.a.) and is thus different from the designs proposed in ref. ${ }^{[20]}$. The parameters that can be varied to optimize the performance of the cavity are the slab thickness, $h$, the slot width, $l$, the waveguide width $W$, the radius of the air holes, $r$, and the hole displacement $d$. The lattice constant of the $\mathrm{PhC}, a$, is set to $620 \mathrm{~nm}$ and can be adjusted so that the cavity resonance precisely meets the target wavelength since all the photonic crystal parameters can be rescaled. To ensure single-mode operation at wavelengths near $\lambda=1600 \mathrm{~nm}$, a slab thickness $h$ of $360 \mathrm{~nm}$ is chosen. A slot width of $135 \mathrm{~nm}$ allows a high confinement of the light in the slot while being easily fabricated. Indeed, narrower $75 \mathrm{~nm}$-wide slots have also been simulated and give similar results in terms of quality factor and modal volume. As in silicon, ${ }^{[19]}$ the best $\mathrm{Q}$ factors are achieved with waveguides larger than the standard W1 waveguide, that is made by removing one row of holes from a perfect photonic crystal. The width of the studied waveguide is $1.15 \sqrt{3}$ a, i.e. 1.15 times the width of a $\mathrm{W} 1$ waveguide. This comes from the necessity to keep the resonating mode in the center of the photonic band gap to get a good lateral confinement and a good quality factor by keeping a theoretical mode well below the light cone. For this set of parameters, we study the quality factor and the modal volume $\mathrm{V}$ as a function of the holes radius of the $\mathrm{PhC}$ and of the hole displacement $d$ that define the cavity. As can be seen on Figure 1.b. that displays the evolution of the quality factor for different hole radius and displacement $d$, a theoretical Q factor up to $0.8 \mathrm{x}$ $10^{6}$ can be expected from these cavities on diamond, a value that is comparable to those reported on silicon. The modal volumes, represented in Figure 1.c., are of the order of $0.15 \lambda_{0}{ }^{3}$ with $\lambda_{0}$ the wavelength in vacuum. This value is two to three times larger than the one achieved in silicon for the same slot widths because of the smaller index contrast and of the resulting lower confinement in diamond $v s$. silicon. Nevertheless, for a typical period of $620 \mathrm{~nm}$, the corresponding sensing volume gets down to a value of 650 attoliter, an extremely small value achieved thanks to the high confinement properties of PhCs.

The mode pattern calculated with a 3D-FDTD simulation of the slotted PhC cavity is shown in

Figure 1.d. It illustrates that the mode profile is well confined in the low refractive index medium, 
Submitted to

thus ensuring an optimized sensitivity to any refractive index change in the slot as well as to any surface modification of the PhC slot. Generally, a measure of the resonant wavelength shift versus the change of the refractive index is the appropriate measure for defining the sensitivity of the optically resonant refractive index sensor. ${ }^{[21]}$ For example, simulations show a change of the resonance wavelength of $580 \mathrm{pm}$ for a refractive index change of the $\mathrm{PhC}$ environment of $10^{-3}$, i.e. a sensitivity of $580 \mathrm{~nm}$ by refractive index unit (RIU). The detection limit of this kind of sensors depends on its capability to accurately measure the spectral shift which in turns depends on the Q factor of the cavity but also on the experimental setup. Following the approach of ref. ${ }^{[21]}$, a rough estimate of the detection limit based on our experimental conditions is $3 \times 10^{-6}$ RIU.
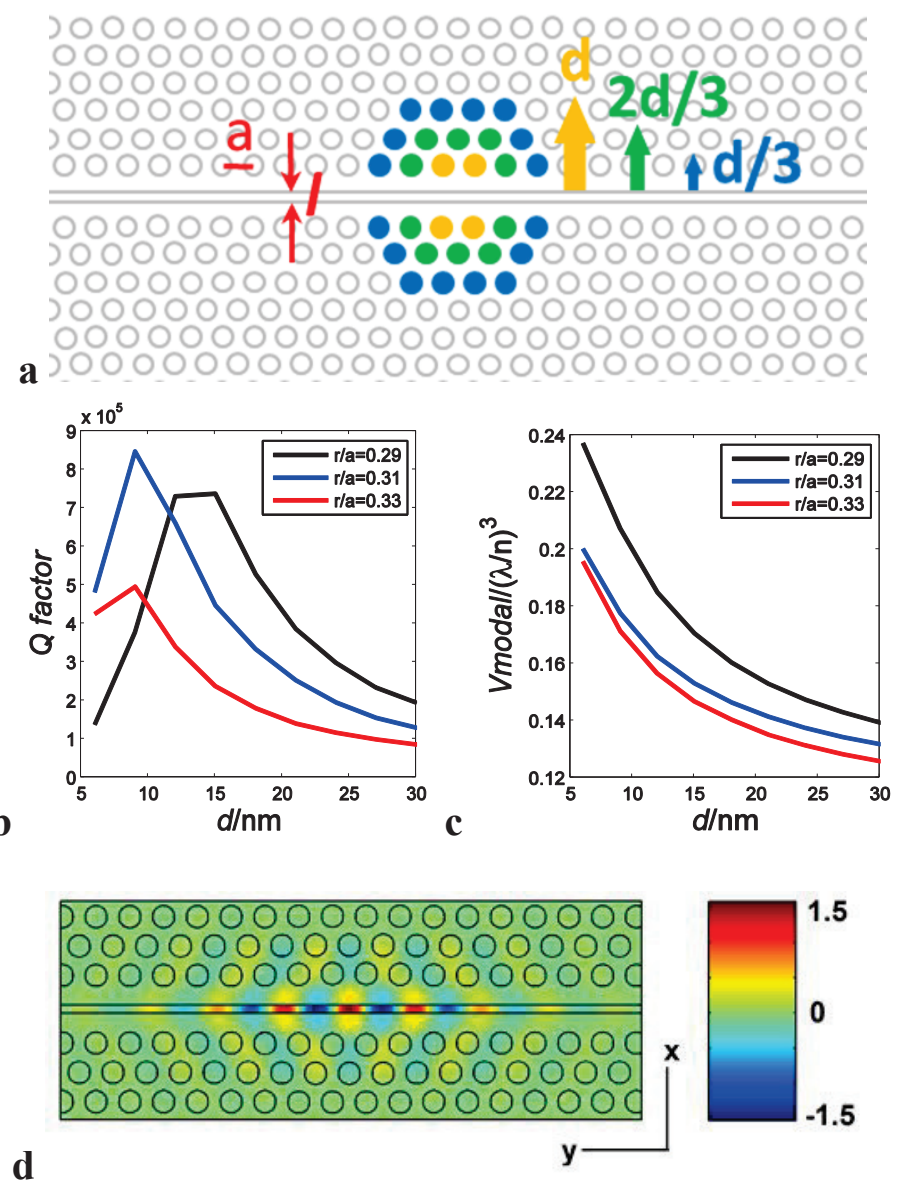

Figure 1. a) Schematic representation of the photonic crystal design with a modulated width cavity and three different hole displacements, a slot width $l$ and a period $a$. b) Simulated Q factors (3DFDTD) of the cavities as a function of hole displacements $d$ for different normalized radii $r / a$. c) Simulated modal volumes of the cavities in units of $(\lambda / n)^{3}$, with $n=1$ (air), as a function of hole displacements $d$ for different normalized radii $r / a$. d) Simulation of slotted photonic crystal air 
cavity showing Ey component of mode profile at cavity resonant wavelength $\lambda=1550 \mathrm{~nm}$, arbitrary unit.

\subsection{Fabrication and optical characterization of the PhCs}

Slotted nanocrystalline diamond PhCs on an integrated photonic platform were fabricated according to the previously described structures (see experimental section for details). Figure 2 shows scanning electronic microscope (SEM) views of the $\mathrm{SPhC}$ at the end of the process.

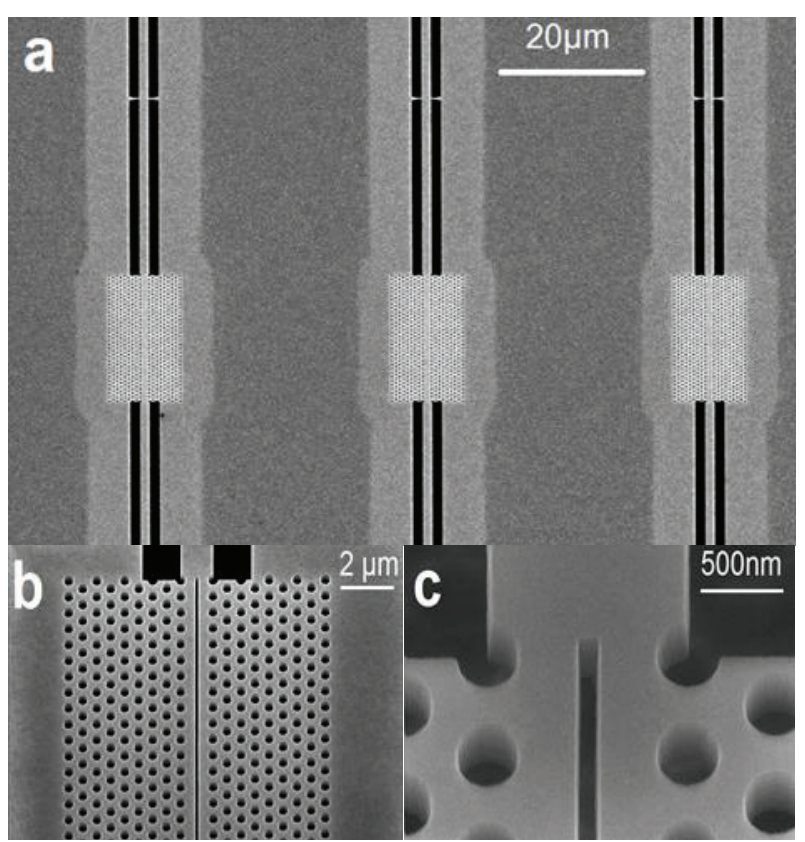

Figure 2. Scanning electron microscope (SEM) views of the fabricated slotted photonic crystal in polycrystalline diamond. a) SEM view of three photonic crystal cavities with their access waveguides maintained by nanotethers. b) Tilted view $\left(45^{\circ}\right)$ of a $\mathrm{PhC}$ and its access waveguide with the slot at the center. c) Tilted close view of a $\mathrm{PhC}$ with the $135-\mathrm{nm}$ wide slot and its access waveguide.

Figure 2.a. is a top view of three sPhCs displaying their access waveguide maintained by nanotethers. These $250-\mu \mathrm{m}$ long waveguides are terminated by inverted tapers (not shown here) that allow an efficient in-plane light injection from a microscope objective or an optical fiber. ${ }^{[14,22]}$ The magnification (tilted view at $45^{\circ}$ ) shown in Figure 2.b. and 2.c. reveals slightly tilted air holes sidewalls relatively to the vertical direction. The verticality of holes is estimated to be better than $3^{\circ}$, indicating a good quality of the diamond etching process that is enough to reach very high $\mathrm{Q}$ factors 
Submitted to

since it has been shown that such an angle theoretically allows for quality factors up to $3 \times 10^{6}$ in silicon PhC cavities. ${ }^{[23]}$

Figure 3 presents an example of the experimental transmission of a $\mathrm{PhC}$ with a period $\mathrm{a}=630 \mathrm{~nm}$ and a slot width of $75 \mathrm{~nm}$.

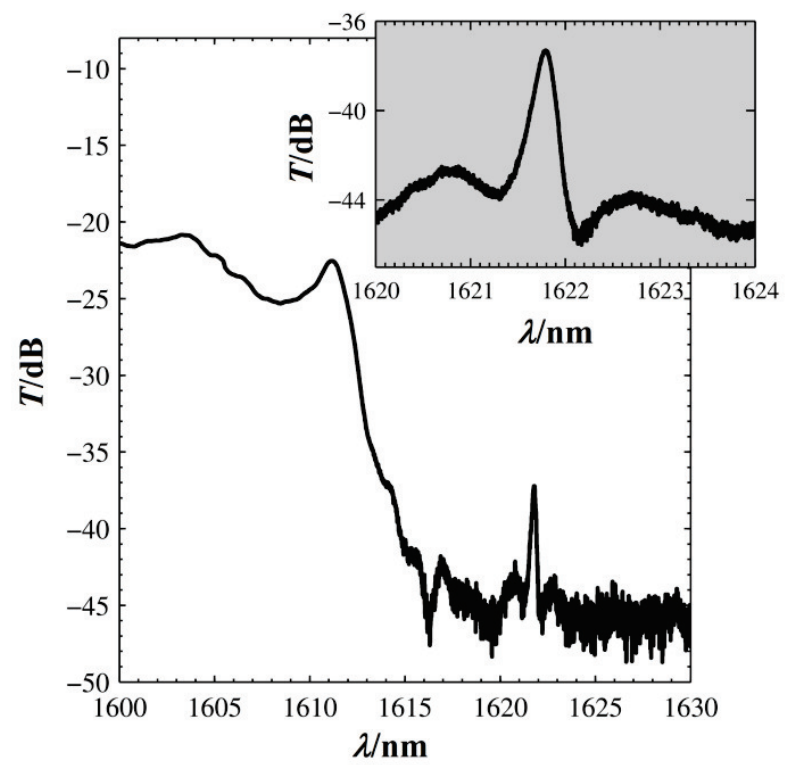

Figure 3. Fiber-to-fiber transmission $(T)$ spectrum of a slotted $\mathrm{PhC}$ with a $\mathrm{Q}$ factor of 6500 . Inset: zoom on the resonance.

The photonic crystal acts as a waveguide for wavelengths shorter than $1615 \mathrm{~nm}$, as confirmed from the observation of the emitted light from the surface, here observed using an infrared camera on Figure 4.a., and a fiber-to-fiber transmission mean value of $-23 \mathrm{~dB}$ is measured. The TE photonic band gap in the diamond $\mathrm{PhC}$ waveguide is seen for wavelengths longer than $1622 \mathrm{~nm}$ where the transmission remains below $-45 \mathrm{~dB}$. The corresponding optical image at this wavelength is shown on Figure 4.b. Finally, the transmission peak at $1621.8 \mathrm{~nm}$ is clearly associated with the cavity mode as presented on Figure 4.c.

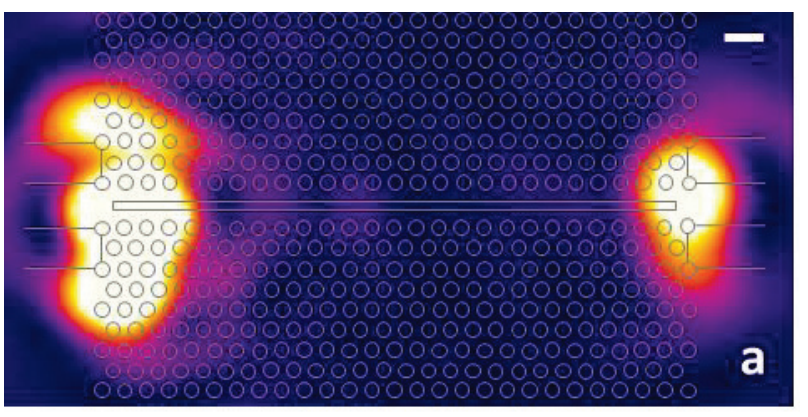




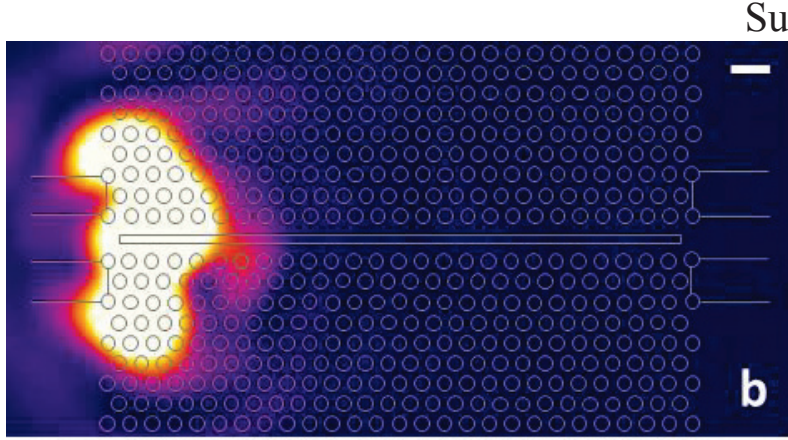

Submitted to

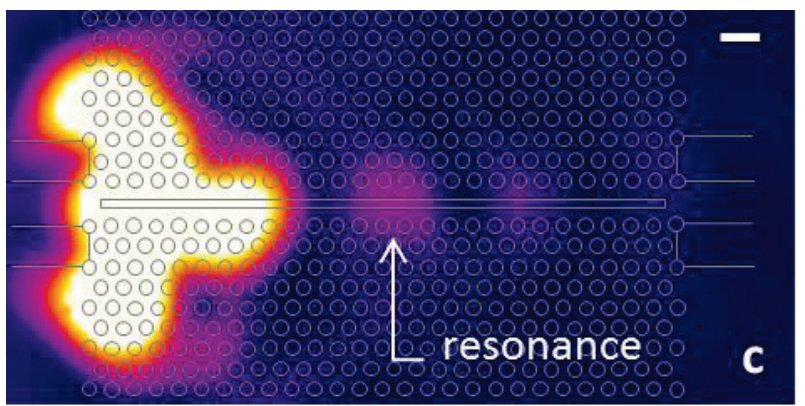

Figure 4. Optical images of the cavity at different wavelengths in false colors. For clarity, a schematic representation of the $\mathrm{PhC}$ is superimposed. a) waveguide transmission. b) cut off in the photonic band gap. c) cavity mode resonating at $1621.8 \mathrm{~nm}$. Scale bar : $1 \mu \mathrm{m}$.

The measured quality factor for this $\mathrm{sPhC}$ reaches 6500 . Regarding other diamond-based photonic devices, the value measured here for our polycrystalline diamond-based $\mathrm{PhC}$ is one among the highest Q factors reported in the literature, ${ }^{[13,24]}$ and can be even compared with PhCs processed from high quality monocristalline diamond. ${ }^{[12,25]}$ The discrepancy of several orders of magnitude between the simulated value and the experimental Q factor is usual in silicon and is generally attributed to fabrication imperfections and surface roughness. Light scattering at grain boundaries in polycristalline diamond is another source of losses that occurs here and does not exist in silicon PhCs nor in the PhCs made in mono-crystalline diamond. Future work on polycristalline diamond with different grain size will clearly identify the quantitative impact of scattering on $\mathrm{Q}$ factors. Nevertheless, the Q factor achieved is only twice lower than those achieved in silicon for similar designs of slotted cavities. ${ }^{[26]}$ Compared to the diamond $\mathrm{PhC}$ cavities we reported in previous works, ${ }^{[14]}$ the introduction of a slot at the center did not affect the Q factor of the cavity significantly. The quality of the structure has even been significantly optimized, mainly through the use of a nitride mask instead of a silica one resulting in a better quality etching (see methods). These Q 
factor values are of the same order as those used on silicon to realize molecular detection at the $\mathrm{PhC}$ surface. $^{[6]}$

\subsection{Characterization of the chemical modifications}

As described above, the slotted polycrystalline diamond-based $\mathrm{PhC}$ exhibits a $\mathrm{Q}$ factor and a design which are both well adapted to probe the effect of a chemical surface modification. A diamond surface is known to be a versatile platform toward functionalization, thanks to its carbon-related chemistry. In this study, we intentionally focus on the simplest surface modifications to probe the sensitivity of the diamond slotted $\mathrm{PhC}$ cavity and the effects of these surface terminations on the PhC optical properties. Hydrogenated and oxidized terminations are also the base of the most common surface functionalization performed on diamond-based devices. For instance, biological moieties such as nucleic acids or proteins can covalently be grafted by their amino groups onto diamond devices, either through an amidation process starting from carboxylic groups ${ }^{[27]}$ or through the direct amine grafting onto an hydrogenated surface. ${ }^{[28]}$ Such surface terminations can be easily conferred to diamond by physico-chemical treatments, such as plasma treatments, annealing or photochemistry. Here we have explored the effect of two surface terminations, based on (i) a plasma hydrogenation of the sample, to saturate homogeneously the diamond surface with $\mathrm{C}-\mathrm{H}$ terminations, ${ }^{[29]}$ and (ii) an oxidation step under UV/Ozone. ${ }^{[30]}$

After each surface treatment, XPS analysis were carried out to investigate the surface modifications induced by the hydrogenation and oxidation treatments. XPS general survey of the hydrogenated diamond reveals that only a small amount (1.4 at.\%) of oxygen remains on the surface, which is partly due to a surface contamination during the transfer between the CVD reactor and the XPS chamber. Note that traces of fluorine are also detected $(0.35$ at.\%) and can be attributed to a contamination during the fabrication process of $\mathrm{PhC}$ as several fluorine-based process are used. After UV/Ozone oxidation, the oxygen quantity increases up to 10.6 at.\%, thus confirming an efficient oxidation of the surface. 


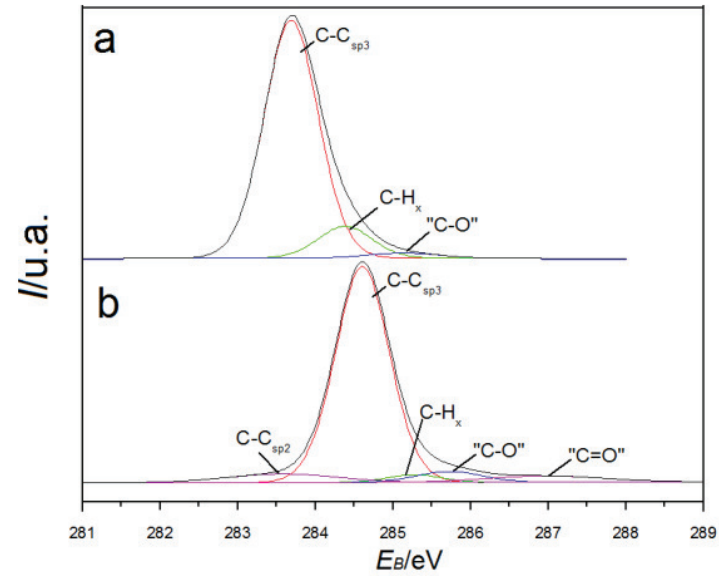

Figure 5. $\mathrm{C}$ 1s core level spectra of a) hydrogenated and b) oxidized diamond layers

Figure 5.a. and b. report the fitted $\mathrm{C} 1 \mathrm{~s}$ core level spectra of the diamond layers after hydrogenation and oxidation treatments respectively. On the hydrogenated sample, the main component attributed to the $\mathrm{C}-\mathrm{C} \mathrm{sp} 3$ diamond bonds is located at $284 \mathrm{eV}$. For an undoped material, this value is a little bit lower than expected, and can be attributed to the superficial conductive layer, which induces a surface band bending, revealed by a downshift on the C1s XPS spectrum, as already evidenced by several authors in the literature. ${ }^{[31,32]}$ Two others components were identified, attributed to $\mathrm{CH}_{\mathrm{x}}$ and etheric bridges, located at +0.7 and $+1.5 \mathrm{eV}$ from the $\mathrm{C}-\mathrm{C}$ sp3 peak, respectively. ${ }^{[33]}$ The component related to the carbon-oxygen groups represent ca. $2 \%$ of the $\mathrm{C} 1 \mathrm{~s}$ core level, which is in agreement with the 1.4 at.\% of oxygen detected on the sample. After oxidation, a strong modification of the C1s core level occurs. First, a strong upshift is observed, with the main component attributed to the C-C sp3 diamond bonds located at $284.9 \mathrm{eV}$, i.e. $+0.9 \mathrm{eV} v$ s. the hydrogenated sample. This shift towards higher binding energies of the $\mathrm{C} 1 \mathrm{~s}$ peak can be related to the loss of the surface conductive layer after the removal of the hydrogenated terminations. This effect has already been evidenced by Ballutaud et al. on equivalent nanocrystalline materials. ${ }^{[32]}$ The proportion of the component related to the etheric bridges increases up to $5 \%$ of the $\mathrm{C} 1 \mathrm{~s}$ core level, while a new component located at $+2.4 \mathrm{eV}$ is identified, attributed to carbonyl functions, and also representing $5 \%$ of the $\mathrm{C} 1 \mathrm{~s}$ core level. At $-1 \mathrm{eV}$, a shoulder is observed, due to the presence of $\mathrm{C}-\mathrm{C}$ sp2 for $5 \%$. A slight surface 
graphitization of the sample seems to occur during the UV/Ozone oxidation, which explains the appearance of this new component.

\subsection{Optical characterization of the modified $\mathrm{sPhC}$}

Prior to any surface modification, measurement of a slotted photonic crystal transmission spectrum just after the end of the process was recorded as a reference. We measured PhC cavities with a period $\mathrm{a}=630 \mathrm{~nm}$, and the structures exhibit a 9-nm shift of the central holes.

a

b
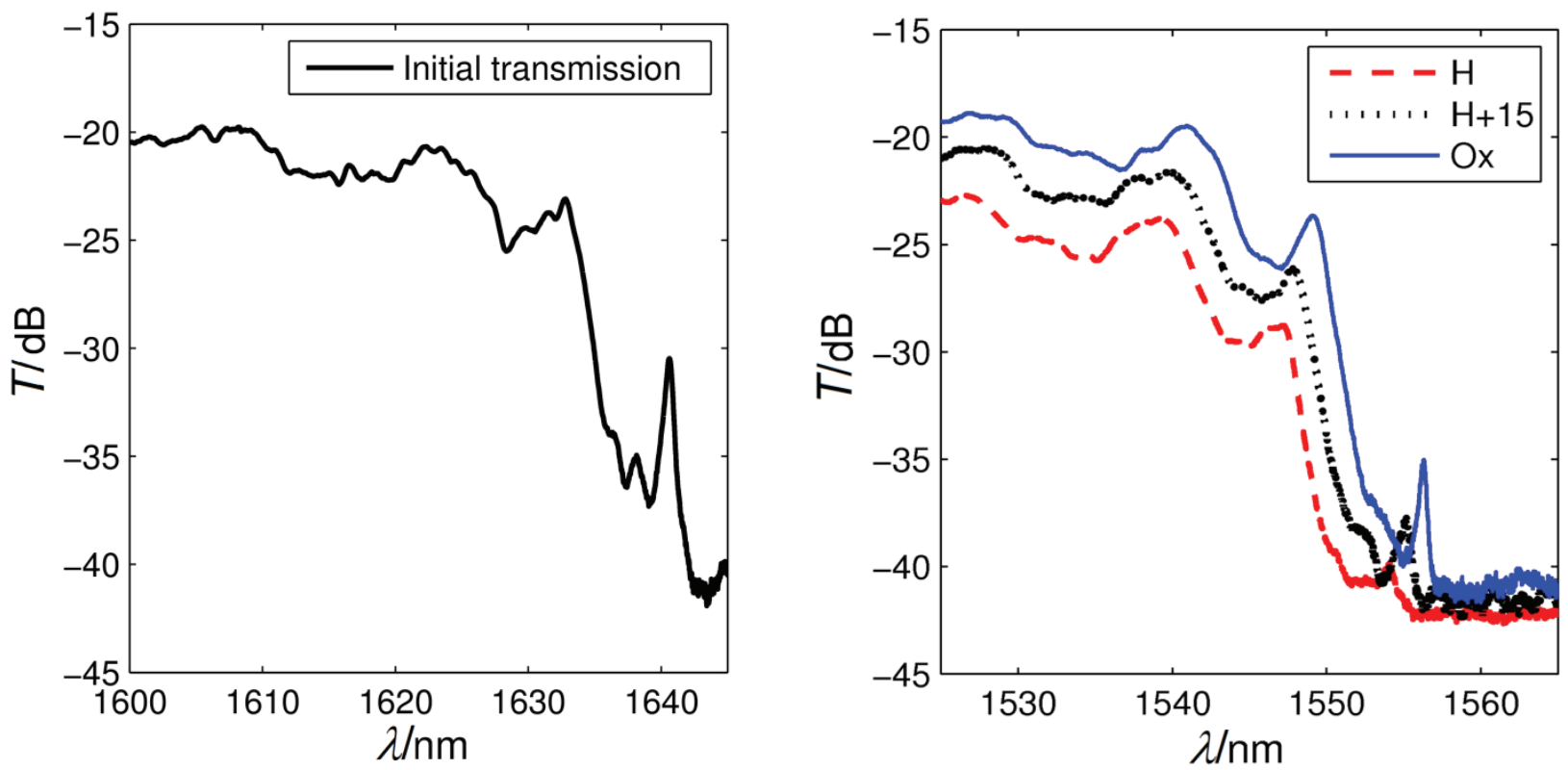

Figure 6. Transmission spectra of the photonic crystal cavities for different surface states. a) Spectrum at the end of the process. b) (dash red) hydrogenated $\mathrm{PhC}-$ (dot black) hydrogenated $\mathrm{PhC}$ after 15 days - (blue) oxidized $\mathrm{PhC}$

Parameters: $\mathrm{a}=630 \mathrm{~nm}, \mathrm{r}=0.31 \mathrm{a}$, slot $=135 \mathrm{~nm}$, barrier length $=8, \mathrm{~d}=9 \mathrm{~nm}$

The transmission spectrum is presented on Figure 6.a. A Q factor of 2000 was measured with a peak resonance at $1640.8 \mathrm{~nm}$. Indeed, we chose a PhC cavity with a shorter barrier in order to have better transmission for the experimental measurements. The coupling coefficient between the access waveguide and the cavity is consequently different and this is why the Q factor is lower (see methods). However, this value remains perfectly adapted for the chemical study as will be shown. We then compared the resonance wavelength and quality factors of the same cavity after different 
Submitted to

successive surface treatments, namely hydrogenation and then oxidation. Figure 6.b. shows the transmission spectra of the same diamond slotted cavity for different surface chemical states. The black curve corresponds to a freshly hydrogenated photonic crystal cavity. As a first observation, we see that the peak resonance is strongly downshifted from 1640.8 to $1554.1 \mathrm{~nm}$ and the Q factor also decreased to 650 , which is three times below the initial value. The downshift of the peak resonance can be attributed to a 6-nm etching occurring during the hydrogenation step, which was confirmed by ellipsometry. Such etching of the surface is inherent to the plasma hydrogenation treatment, but, as shown by the XPS analysis, does not affect the quality of the diamond surface. However, according to the simulations, the Q-factor decrease cannot only be attributed to diamond etching. After ageing for 15 days in air, transmission of the same sample was recorded again and is represented by the red curve on Figure 6.b. Surprisingly, the resonance peak of the PhC cavity presents a red shift of $1.1 \mathrm{~nm}(1555.2 \mathrm{~nm})$ while the $\mathrm{Q}$ factor increases to reach a value of 1050 . Finally, when an intentional surface oxidation is performed on the sample, through the UV/ozone treatment, the red shift continues to occur, with a shift of $+1.1 \mathrm{~nm}(1556.3 \mathrm{~nm})$ and a value of the $\mathrm{Q}$ factor back to the initial one, namely 2000 . The transmission also evolves according to the surface modification. An increase is observed when the sample changes from a hydrogenated state to an oxidized one. It is particularly observable in the allowed transmission frequency range for wavelengths shorter than $1550 \mathrm{~nm}$ and at the cavity resonance frequency.

\subsection{Effect of the chemical modification on $\mathrm{PhC}$ optical properties}

From these experiments, our polycrystalline diamond-based PhCs clearly exhibit a strong dependence on the surface state, with a high sensitivity to the nature of the surface terminations revealed by both resonance peak position and value of the $\mathrm{Q}$ factor. Since the refractive index change at the diamond surface is expected to be very small, the evolution of the resonant frequency and quality factor can be estimated thanks to first order perturbations. Indeed, the shift $\Delta \omega$ of the resonant frequency of a cavity due to a small change $\Delta \varepsilon$ of the dielectric constant $\varepsilon(\vec{r})=n^{2}(\vec{r})$, with 
$n$ the refractive index of the materials making up the cavity, is given with a second order accuracy by Equation (1) :

$$
\Delta \omega=\frac{\omega}{2} \frac{\iiint \Delta \varepsilon(\vec{r})\|\vec{E}(\vec{r})\|^{2} d^{3} \vec{r}}{\iiint \varepsilon(\vec{r})\|\vec{E}(\vec{r})\|^{2} d^{3} \vec{r}}+O\left((\Delta \varepsilon)^{2}\right)
$$

where $\vec{E}(\vec{r})$ is the electric field of the unperturbed resonant mode. ${ }^{[34]} \mathrm{A}$ small absorption, represented by the imaginary part of the refractive index or the dielectric constant, leads to a complex frequency shift. The imaginary part of this shift represents a cavity field decreasing as $e^{-\operatorname{Im}(\Delta \omega t)}$ or in other words a cavity mode with a quality factor of $Q_{a}=\frac{\omega}{2 \operatorname{Im}(\Delta \omega)}$. The total quality factor $Q_{t}$ of the cavity is then given by Equation (2):

$$
\mathrm{Q}_{\mathrm{t}}^{-1}=\mathrm{Qu}^{-1}+\mathrm{Q}_{\mathrm{a}}^{-1}
$$

where $Q_{u}$ is the quality factor of the unperturbed cavity.

Here, the $\vec{E}(\vec{r})$ field of the unperturbed cavity resonant mode has been calculated by 3D-FDTD once and the perturbative approach has been used to calculate the frequency shifts much faster than with a full 3D calculation. However, a few supplementary FDTD calculations have been performed to check that this perturbative approach is accurate enough to explain the experimental results. Considering that when the surface is tuned from hydrogenated to oxidized the chemical modification is of the order of the molecule layer, the refractive index modification at the surface should be effective over a thickness of the order of a few Angstroms, as can be expected with the progressive replacement of a $\mathrm{H}$ termination by a $\mathrm{O}$ termination. However, the expected very small refractive index change resulting from such replacement is not compatible with the frequency shifts observed with the recorded values beyond $2 \mathrm{~nm}$. Obviously, we can also consider that adsorbed molecules or groups of adsorbates, with a thickness of several nm, can be deposited at the surface. Then the frequency shift can reach the $2 \mathrm{~nm}$ observed. However, if such an hypothesis turned out to be true, then the Q factor would not increase as observed, because these adsorbates cannot bring 
optical gain. Actually, the initial value is recovered, which means that there is an intern phenomenon related to the properties of the layer and that it is not just a chemical effect.

To explain this phenomenon, we have to get back to the transfer doping model. ${ }^{[35]}$ It is well known that hydrogenated terminations confer to diamond films specific electronic properties. ${ }^{[36]}$ Diamond surface becomes conductive when hydrogenated. The creation of a surface conductive layer (SCL) due to transfer doping has already been thoroughly described in the literature, and is known to disappear when the surface is oxidized. Maier and coworkers presented about a decade ago the associated mechanism. ${ }^{[35]}$ The extension of this conductive layer is usually considered to be between 5 and $30 \mathrm{~nm}$ from the diamond surface. ${ }^{[37]}$ Such a layer may affect the optical properties of the diamond surface though it has never been characterized so far. Indeed, the absorption phenomenon inherent to such conductive materials would be consistent with the experimental evolution of our quality factor. When the surface is freshly hydrogenated, the surface conductivity is high as well as the loss and the imaginary part of the refractive index. The surface loses partly its conductivity when aged in air, ${ }^{[38]}$ as hydrogenated diamond surfaces are known to be sensitive to air exposure, with a limited but effective spontaneous surface oxidation, with the creation of oxidized terminations among the hydrogenated ones. ${ }^{[39]}$ Then, the conductivity completely drops down when the surface is oxidized by $\mathrm{UV} / \mathrm{O}_{3}$ treatment, which induces the fall of the imaginary part of the refractive index. Note that the rise of the transmission is also consistent with the continuously disappearing surface conductivity since the losses due to the propagation along the hydrogenated access waveguides and $\mathrm{PhC}$ structure decrease. The transmission of the $\mathrm{PhC}$ at the resonance frequency can also be attributed to the cavity losses but the transmission measurement of the PhC, as opposed to the quality factor measurement, is affected by uncertainties such as the evolution of the coupling efficiency and access waveguide transmission in hydrogenated diamond.

For a given thickness of the surface conductive layer, we can relate the resonant wavelength shift and the evolution of the $\mathrm{Q}$ factor to the refractive index of the surface conductive layer using Equation (1). All the surface of the diamond $\mathrm{PhC}$ is supposed to be hydrogenated as confirmed by 
XPS analysis. The real and imaginary part of the refractive index of the SCL can then be calculated as a function of the SCL thickness from the experimentally measured wavelengths shift and Q factors (Figure 7).

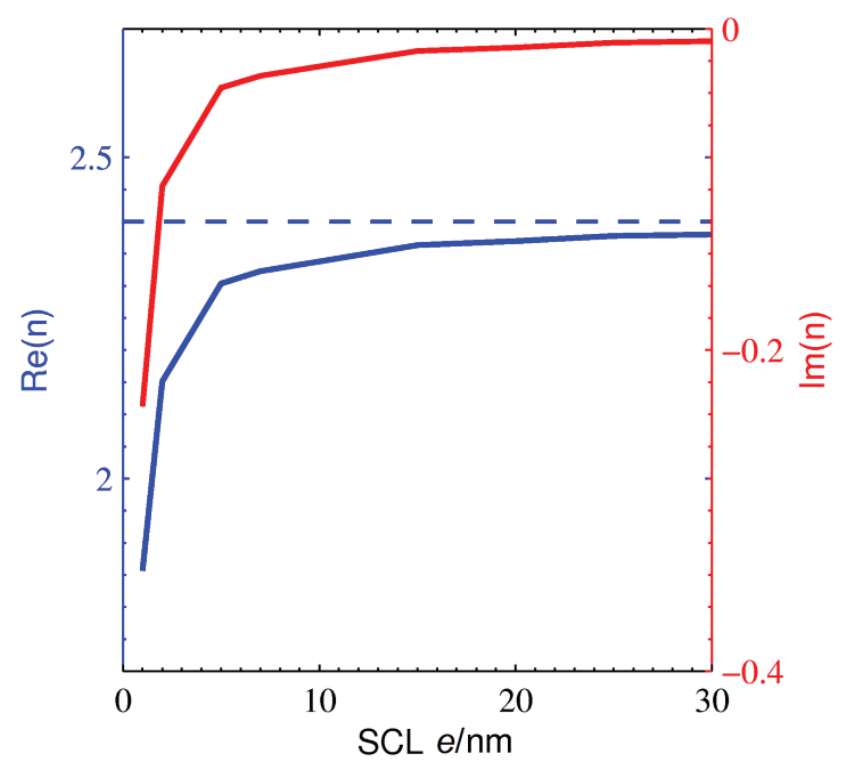

Figure 7. Evolution of the real and imaginary parts of the surface conductive layer of hydrogenated diamond as a function of the thickness of this layer $(e)$.

Because the wavelength shift depends on the volume of the region of modified refractive index probed by the electromagnetic field as seen in Equation (1), its measure only gives us information on the product of the refractive index change by the thickness of the SCL. Nevertheless, for a typical average SCL thickness of $15 \mathrm{~nm}$, the refractive index calculated from the measurements is equal to $2.36-0.014 \mathrm{i}$. As can be seen, the refractive index change is in the order of $2 \%$ and even smaller if the SCL spreads over several tens of nanometers. Considering that with a moderate quality factor of 2000 , wavelength shifts of the resonance as small as $0.1 \mathrm{~nm}$ can be easily observed, refractive index changes of less than $0.1 \%$ that spread over $15 \mathrm{~nm}$ in the diamond layer can be detected with this kind of photonic crystal. The imaginary part of the refractive index is equivalent to propagation losses of the order of $1000 \mathrm{~cm}^{-1}$. Even if the thickness of the conductive layer is small, this value can be considered very high in the perspectives of diamond photonic circuits where ultra-high quality factor cavities and low loss waveguide are required. As a consequence, such 
hydrogenated surfaces may be a limitation for sensing using PhCs as the detection limit would be reduced. Indeed, the conduction phenomenon will still occur on a functionalized surface and, unless the sensing property specifically addresses this surface conductivity property as for diamond-based ion sensitive field effect transistors, ${ }^{[40]}$ it may be a limitation. However, the geometry used here largely compensates this, and one could expect that it would still be perfectly adapted for real-time detection measurements. Anyway, a full oxidation of the diamond surface, for example using a UV treatment, may thus appear as a prerequisite for the exploitation of diamond photonic devices. As we showed, both stability and efficiency will be higher on oxidized diamond based $\mathrm{PhC}$ than on hydrogenated.

\section{Conclusion}

We have investigated the sensitivity of diamond slotted cavities in photonic crystals to chemical surface modifications. We demonstrate that the optical properties of the diamond $\mathrm{shC}$ are highly affected after simple surface treatments and namely hydrogenation and oxidation. We observed that a resonance wavelength shift of the cavity occurs and is accompanied by a decrease of the Q factor when the surface chemistry is simply turned from oxidized to hydrogenated. Interestingly, this phenomenon is reversible as the initial values of the $\mathrm{Q}$ factor are recovered when the surface is reoxidized. This result can be attributed to an intern phenomenon related to a property of the material, that is the apparition of a conductive layer at the surface when diamond is hydrogenated. This electronic modification of the material strongly modifies the optical features of diamond $\mathrm{sPhCs}$. Since these characteristics are directly depending of the optical environment, the use of diamond slotted $\mathrm{PhC}$ is an effective though indirect approach to get an estimate of the refractive index of the surface conductive layer. These experimental results give a strong indication of the sensitivity of slotted PhC cavity in diamond since it can detect the refractive index changes induced by surface chemistry. As a matter of fact, these results are very promising for the realization of very sensitive sensors based on diamond $\mathrm{PhC}$ with specific functionalization. 


\section{ADVANCED \\ Submitted to \\ MATERIALS}

\section{Experimental Section.}

\subsection{Fabrication process}

\subsubsection{Diamond layer preparation}

For this study, we have used nanocrystalline diamond films with a columnar structure exhibiting a thickness of $400 \mathrm{~nm}$ deposited on two-inch Si (100) substrates wafers. They were grown at CEALIST using chemical vapor deposition assisted by microwave plasma (MPCVD) with a diamond nanoseeding treatment. The deposition was performed under $35 \mathrm{hPa}$ pressure using methane diluted in hydrogen $\left(0.6 \% \mathrm{CH}_{4} / \mathrm{H}_{2}\right)$ at a total flow of $250 \mathrm{sccm}$. The microwave power was $1100 \mathrm{~W}$ and the substrate was held at a temperature of $1123 \mathrm{~K}$. The growth rate was $220 \mathrm{~nm} / \mathrm{h}$. ${ }^{[41]}$ The root mean square (RMS) roughness of the 400-nm thick resulting diamond films is around $10 \mathrm{~nm}$ with a peak to valley roughness that can reach $80 \mathrm{~nm}$. This roughness prevents the use of e-beam lithography and the achievement of high quality factor cavities due to the strong scattering at the surface. To smooth the diamond layer, a 200-nm thick hydrogen silsesquioxane (HSQ) layer is first spin-coated on the rough polycrystalline diamond layer and baked on a hot plate. This results in a smooth surface silica-like layer on top of the sample. The layers are then etched using an inductively coupled plasma (ICP) reactive ion etching with oxygen and argon. A 360-nm layer of smooth diamond is finally obtained. Silica residues are removed in hydrofluoric acid. The RMS roughness can reach $1 \mathrm{~nm}$ only on a $10 \mathrm{x} 10 \mu \mathrm{m}^{2}$ area, with a peak to valley roughness below $10 \mathrm{~nm}$.

\subsubsection{PhC fabrication}

The whole fabrication process consists of the following standard steps: a 65-nm thick layer of silicon nitride is first deposited by plasma enhanced chemical vapor deposition (PECVD) on the top of the smoothed diamond layer. Then, e-beam resist is spin-coated on the nitride layer. The photonic crystal and the access waveguide are patterned in the resist using electronic lithography. Using an ICP reactive ion etching (RIE), the pattern is transferred from the resist to nitride and then from nitride to diamond. The remaining nitride mask is removed in hydrofluoric acid. Finally, to fabricate 
a free-standing diamond membrane the silicon substrate is partially etched using a vapor phase isotropic $\mathrm{XeF}_{2}$ etching process.

\subsection{Optical characterization}

Light from a tunable laser source with a 1-pm resolution $(1500-1680 \mathrm{~nm}$, output power $30 \mu \mathrm{W})$ is injected and collected into the access waveguide with the use of two polarization maintaining lensed optical fibers with spot diameter of $\sim 2.75 \mu \mathrm{m}$. Light from the output of a waveguide is then sent to a polarizer to keep only the TE component of the field (E field parallel to the plane of the $\mathrm{PhC}$ ) as in ref. $^{[42]}$. The transmitted power is recorded with a photodiode and analyzed with an oscilloscope. For cavity measurements, the wavelength sweep on the oscilloscope was limited to the region around the resonance to maximize the resolution.

Considering that the photonic crystal waveguide acts as a barrier for the cavity mode, and the length of this barrier determines the coupling of the cavity to the ridge access waveguides as well as the loaded quality factor of the cavity, $\mathrm{PhC}$ with different parameters are chosen. A PhC cavity with a long barrier is expected to reach the highest $\mathrm{Q}$ factor, while a $\mathrm{PhC}$ with a short barrier allows a better guidance and transmission of light, which is more advantageous for sensing applications.

\subsection{Surface treatments}

Hydrogenation was achieved using a microwave CVD plasma treatment for 30 minutes. Parameters were tuned with a pressure of $40 \mathrm{hPa}$ of $\mathrm{H}_{2}$. The microwave power was adjusted to $1 \mathrm{~kW}$ in order to reach a temperature of $1023 \mathrm{~K}$ measured in situ using an optical pyrometer (Ircon Modline 3 series 3L). Oxidation was achieved by a UV exposure (OSRAM XERADEX) for 2 hours at $250 \mathrm{hPa}$ under air on samples previously hydrogenated. The chemical surface state of the treated samples was characterized by X-ray Photoelectron Spectroscopy. The dimensions and the difficulty to properly evacuate the charges on free diamond membranes such as the $\mathrm{PhC}$ prevent from a direct characterization of them by XPS. Therefore, spectroscopic characterizations were performed on reference samples. These samples are extracted from the smoothed diamond layer used for the fabrication of $\mathrm{PhC}$ which were thus exposed to the same treatments. The surface chemistry was 
Submitted to

monitored with a monochromatized Al Ka anode $(1486.6 \mathrm{eV})$, calibrated versus the $\mathrm{Au} 4 \mathrm{f} 7 / 2$ peak located at $84.0 \mathrm{eV}$. Areas of XPS core levels were extracted after a Shirley correction of the background. Carbon and oxygen contents are calculated from XPS spectra after correction by the photoionization cross-sections. A curve fitting procedure was performed to extract the components in the $\mathrm{C} 1 \mathrm{~s}$ spectra. Voigt functions were used with a Lorentzian width of $0.4 \mathrm{eV}$ for $\mathrm{C} 1 \mathrm{~s}$.

\section{Acknowledgements}

C. Blin would like to thank the financial support from the French Atomic Energy Commission. X. Checoury gratefully acknowledges CEA for the support provided for his "Chaire CEA/Université Paris Sud". This work was also supported by the French RENATECH network and Conseil général de l'Essonne. Authors thank J-C. Arnault for providing facilities and support with XPS recordings.

Received: ((will be filled in by the editorial staff))

Revised: ((will be filled in by the editorial staff)) Published online: ((will be filled in by the editorial staff))

\section{References}

[1] J. Homola, Chem. Rev. 2008, 108, 462.

[2] C. a. Barrios, M. J. Bañuls, V. González-Pedro, K. B. Gylfason, B. Sánchez, A. Griol, a. Maquieira, H. Sohlström, M. Holgado, R. Casquel, Opt. Lett. 2008, 33, 708.

[3] E. Kuramochi, M. Notomi, S. Mitsugi, A. Shinya, T. Tanabe, T. Watanabe, Appl.Phys. Lett. 2006, 88 .

[4] B. S. Song, S. Noda, T. Asano, Y. Akahane, Nat.Mater.2005, 4, 207.

[5] E. Chow, A. Grot, L. W. Mirkarimi, M. Sigalas, G. Girolami, Opt. Lett. 2004, 29, 1093.

[6] M. G. Scullion, A. Di Falco, T. F. Krauss, Biosensors \& bioelectronics 2011, 27, 101.

[7] a) M. Lee, P. M. Fauchet, Optics Express 2007, 15, 4530; b) N. Skivesen, A. Têtu, M. Kristensen, J. Kjems, L. H. Frandsen, P. I. Borel, Optics Express 2007, 15, 3169; c) S. Pal, A. R. Yadav, M. A. Lifson, J. E. Baker, P. M. Fauchet, B. L. Miller, Biosensors and Bioelectronics 2013, 44, 229.

[8] M. Borselli, T. J. Johnson, O. Painter, Appl. Phys. Lett. 2006, 88, 131114.

[9] a) R. C. Major, X.-Y. Zhu, Langmuir 2001, 17, 5576; b) Z. Lin, T. Strother, W. Cai, X. Cao, L. M. Smith, R. J. Hamers, Langmuir 2002, 18, 788. 
Submitted to

[10] a) M. Liao, S. Hishita, E. Watanabe, S. Koizumi, Y. Koide, Adv. Mater. (Deerfield Beach, Fla.) 2010, 22, 5393; b) O. a. Williams, Diam. and Rel. Mater. 2011, 20, 621; c) M. Dankerl, M. V. Hauf, M. Stutzmann, J. a. Garrido, Phys. Status Solidi (a) 2012, 209, 1631. d) P. Bergonzo, A. Bongrain, E. Scorsone, A. Bendali, L. Rousseau, G. Lissorgues, P. Mailley, Y. Li, T. Kauffmann, F. Goy, IRBM 2011, 32, 91;

[11] I. Aharonovich, A. D. Greentree, S. Prawer, Nat. Photon. 2011, 5, 397.

[12] A. Faraon, C. Santori, Z. Huang, V. M. Acosta, R. G. Beausoleil, Phys. Rev. Lett. 2012, 109, 2.

[13] B. J. M. Hausmann, I. B. Bulu, P. B. Deotare, M. McCutcheon, V. Venkataraman, M. L. Markham, D. J. Twitchen, M. Lončar, Nano Lett. 2013, 13, 1898.

[14] X. Checoury, D. Néel, P. Boucaud, C. Gesset, H. Girard, S. Saada, Appl. Phys. Lett. 2012, 171115,1 .

[15] a) A. Hartl, E. Schmich, J. A. Garrido, J. Hernando, S. C. R. Catharino, S. Walter, P. Feulner, A. Kromka, D. Steinmuller, M. Stutzmann, Nat. Mater. 2004, 3, 736; b) B. Sun, S. E. Baker, J. E. Butler, H. Kim, J. N. Russell, L. Shang, K.-Y. Tse, W. Yang, R. J. Hamers, Diam. and Rel. Mater. 2007, 16, 1608; c) W. Yang, O. Auciello, J. E. Butler, W. Cai, J. A. Carlisle, J. E. Gerbi, D. M. Gruen, T. Knickerbocker, T. L. Lasseter, J. N. Russell, L. M. Smith, R. J. Hamers, Nat. Mater. 2002, 1, 253.

[16] a) S. Tomljenovic-Hanic, M. J. Steel, C. M. de Sterke, J. Salzman, Optics Express 2006, 14, 3556; b) I. Bayn, J. Salzman, Eur. Phys. J. Appl. Phys. 2007, 37, 19; c) C. Kreuzer, J. Riedrich-Möller, E. Neu, C. Becher, Opt. Express 2008, 16, 1632; d) S. Tomljenovic-Hanic, A. D. Greentree, C. M. de Sterke, S. Prawer, Opt. Express 2009, 17, 6465; e)T. M. Babinec, J. T. Choy, K. J. M. Smith, M. Khan, M. Loncar, J. of Vac. Sci. \& Tech. B 2011, 29, 10601

[17] C. F. Wang, R. Hanson, D. D. Awschalom, E. L. Hu, T. Feygelson, J. Yang, J. E. Butler, Appl. Phys. Lett. 2007, 91, 201112.

[18] M. P. Hiscocks, C.-H. Su, B. C. Gibson, A. D. Greentree, L. C. L. Hollenberg, F. Ladouceur, Opt. Express. 2009, 17, 7295.

[19] T. Yamamoto, M. Notomi, H. Taniyama, E. Kuramochi, Y. Yoshikawa, Y. Torii, T. Kuga, Opt. Express 2008, 16, 13809.

[20] a) S.-H. Kwon, T. Sunner, M. Kamp, A. Forchel, Opt. Express 2008, 16, 11709; b) A. Di Falco, L. O. Faolain, T. F. Krauss, Appl. Phys. Lett. 2009, 063503, 6; c) J. Jagerska, H. Zhang, Z. Diao, N. Le Thomas, R. Houdré, Opt. Lett. 2010, 35, 2523.

[21] I. M. White, X. Fan, Opt. Express 2008, 16, 1020. 